Taxonomía y sistemática

\title{
Sacoglosos (Opisthobranchia: Sacoglossa) del estado Nueva Esparta y Los Frailes, Venezuela
}

\author{
Sacoglossans (Opisthobranchia: Sacoglossa) from Nueva Esparta state and Los Frailes, Venezuela \\ Makcim de Sisto, Roberta Crescini*, William Villalba, Beatriz Ríos, \\ Verónica Gómez e Yennimar Mata \\ Escuela de Ciencias Aplicadas del Mar, Universidad de Oriente, Boca del Río, Isla de Margarita, Venezuela
}

Recibido el 27 de marzo de 2015; aceptado el 3 de noviembre de 2015

Disponible en Internet el 2 de marzo de 2016

\begin{abstract}
Resumen
Se presenta una lista sistemática de 7 especies de gasterópodos sacoglosos recolectadas desde marzo de 2013 hasta enero de 2014 en el estado Nueva Esparta y Los Frailes. En dicha lista, Elysia serca Marcus, 1955 y Elysia cauze Marcus, 1957 se registran por primera vez en Venezuela. Adicionalmente, se propone una clave de identificación para estos animales.

Derechos Reservados @ 2016 Universidad Nacional Autónoma de México, Instituto de Biología. Este es un artículo de acceso abierto distribuido bajo los términos de la Licencia Creative Commons CC BY-NC-ND 4.0.
\end{abstract}

Palabras clave: Gastropoda; Sistemática; Taxonomía; Primer registro; Caribe sur

\begin{abstract}
A systematic list including 7 species of sacoglossan gastropods collected between March 2013 and January 2014, from Nueva Esparta state and Los Frailes is herein presented. In this list, Elysia serca Marcus, 1955 and Elysia cauze Marcus, 1957 are recorded for the first time in Venezuela. In addition, an identification key for these animals is proposed.

All Rights Reserved (C) 2016 Universidad Nacional Autónoma de México, Instituto de Biología. This is an open access item distributed under the Creative Commons CC License BY-NC-ND 4.0.

Keywords: Gastropoda; Systematics; Taxonomy; First record; Southern Caribbean
\end{abstract}

\section{Introducción}

Los opistobranquios son un grupo de moluscos marinos que exhiben un amplio arreglo de formas corporales, por lo que su anatomía externa tiene muy diversos aspectos (Ardila, Báez y Valdés, 2007). El arreglo de los sistemas, los cambios en la forma del cuerpo y, en algunas especies, la pérdida de la concha permitieron el desarrollo de muchas otras estructuras externas como ceratas, parapodios y rinóforos, los cuales tienen funciones sensoriales, defensivas o respiratorias (Mikkelsen, 2002).

\footnotetext{
* Autor para correspondencia.

Correo electrónico: robertacrescini@gmail.com (R. Crescini).

La revisión por pares es responsabilidad de la Universidad Nacional Autónoma de México.
}

Entre los distintos grupos que conforman los opistobranquios, los sacoglosos son babosas herbívoras (Ardila et al., 2007) que se alimentan del citoplasma de las células de algas, fanerógamas y/o diatomeas, en algunos casos reteniendo en su interior los cloroplastos funcionales -cleptoplastia-, de los cuales obtienen productos fotosintéticos y metabolitos secundarios (Maeda, Kajida, Maruyama y Hirano, 2010), por lo que su distribución está limitada a la zona fótica (Jensen, 2007). El orden comprende 250-300 especies aproximadamente, las cuales se caracterizan por ser de talla pequeña, con o sin concha, de color usualmente verde, siendo su patrón de coloración igual o parecido al del alga de la que se alimenta -homocromía nutricional-. Además, cuentan con la presencia de un «asca», el cual es un saco epitelial que almacena los dientes radulares usados (Jensen, 1997).

Muchos autores han trabajado con la taxonomía de los opistobranquios en el mar Caribe y el Atlántico: entre las publicaciones 
más conocidas están las de Marcus y Marcus (1960), quienes estudiaron a los opistobranquios en el Atlántico americano; Marcus y Marcus (1967) describen las especies del Caribe sur; Edmunds (1964) y Thompson $(1977 ; 1980)$ estudiaron los opistobranquios de Jamaica; Edmunds y Just (1983) describen los nudibranquios de Barbados; Valdés, Hamann y Dupont (2006) realizan una guía ilustrada de los opistobranquios del sur del mar Caribe; Ortea, Espinosa, Buske y Caballer (2013) y Ortea, Espinosa, Caballer y Buske (2012) realizan inventarios de opistobranquios de Guadalupe. En Sacoglossa destacan los trabajos focalizados en defensa química y metabolitos secundarios (Cimino y Ghiselin, 1999), evolución y biogeografía (Jensen, 1997, 2007), y descripciones de especies (Caballer, Ortea y Espinosa, 2006; Ortea y Espinosa, 2002; Ortea, Moro y Espinosa, 1997; Ortea, Moro, Bacallado y Espinosa, 1998), entre otros.

En Venezuela, las investigaciones acerca de los opistobranquios y de los sacoglosos han sido escasas (De Sisto, 2014; Grune, Crescini, de Sisto, Velásquez y Villalba, 2014): en el caso particular de los opistobranquios, en general, se han enfocado en publicar nuevas citas para el país (Crescini, Villalba y de Sisto, 2013; Mariño, Farfan y Caballer, 2011) o en la descripción de nuevas especies (Caballer y Ortea, 2012, 2015; Crescini, de Sisto y Villalba, 2013). En sacoglosos destacan los trabajos de Gavagnin, Mollo, Cimino y Ortea (1996), Gavagnin et al. (1997) y Gavagnin, Mollo, Montanaro, Ortea y Cimino (2000), quienes estudian aspectos químicos y ecológico de Elysia crispata, y el catálogo de opistobranquios y sacoglosos de Caballer (2007), quien cita Placida verticillata para la isla de Margarita. La presencia de estos organismos en inventarios generales de moluscos en ciertas zonas del país ha producido, a su vez, algunos registros muy difíciles de confirmar por la ausencia de ilustraciones o descripciones detalladas de los mismos (Capelo y Buitrago, 1998; Capelo et al., 2014; Miloslavich et al., 2010). Es por ello que se presenta una lista taxonómica de los sacoglosos del estado Nueva Esparta con el propósito de mejorar y complementar la información existente de las especies.

\section{Materiales y métodos}

El estudio se llevó a cabo durante el periodo comprendido desde marzo de 2013 hasta enero de 2014 en 24 puntos del estado Nueva Esparta $\left(10^{\circ} 44,11^{\circ} 10^{\prime} \mathrm{N}, 63^{\circ} 46^{\prime}, 64^{\circ} 13^{\prime} \mathrm{O}\right)$ (fig. 1), revisando mensualmente algas y praderas de Thalassia testudinum, sustratos a los cuales estos organismos se encuentran asociados. Los organismos fueron recolectados manualmente durante buceo autónomo o en apnea, colocados en envases plásticos pequeños y llevados al laboratorio, donde fueron fotografiados, dibujados, identificados y, posteriormente, preservados en alcohol etílico al 70\%, etiquetados y guardados en la colección del laboratorio de Malacología de la Escuela de Ciencias Aplicadas del Mar bajo el acrónimo MOS y su respectiva numeración. Con la información reunida sobre las características de los ejemplares recolectados se realizó una propuesta de clave sencilla para la identificación de las especies de sacoglosos del estado Nueva Esparta y Los Frailes encontradas en el presente estudio. La clave, identificación y el resumen taxonómico se realizaron siguiendo los criterios establecidos por Marcus (1980).

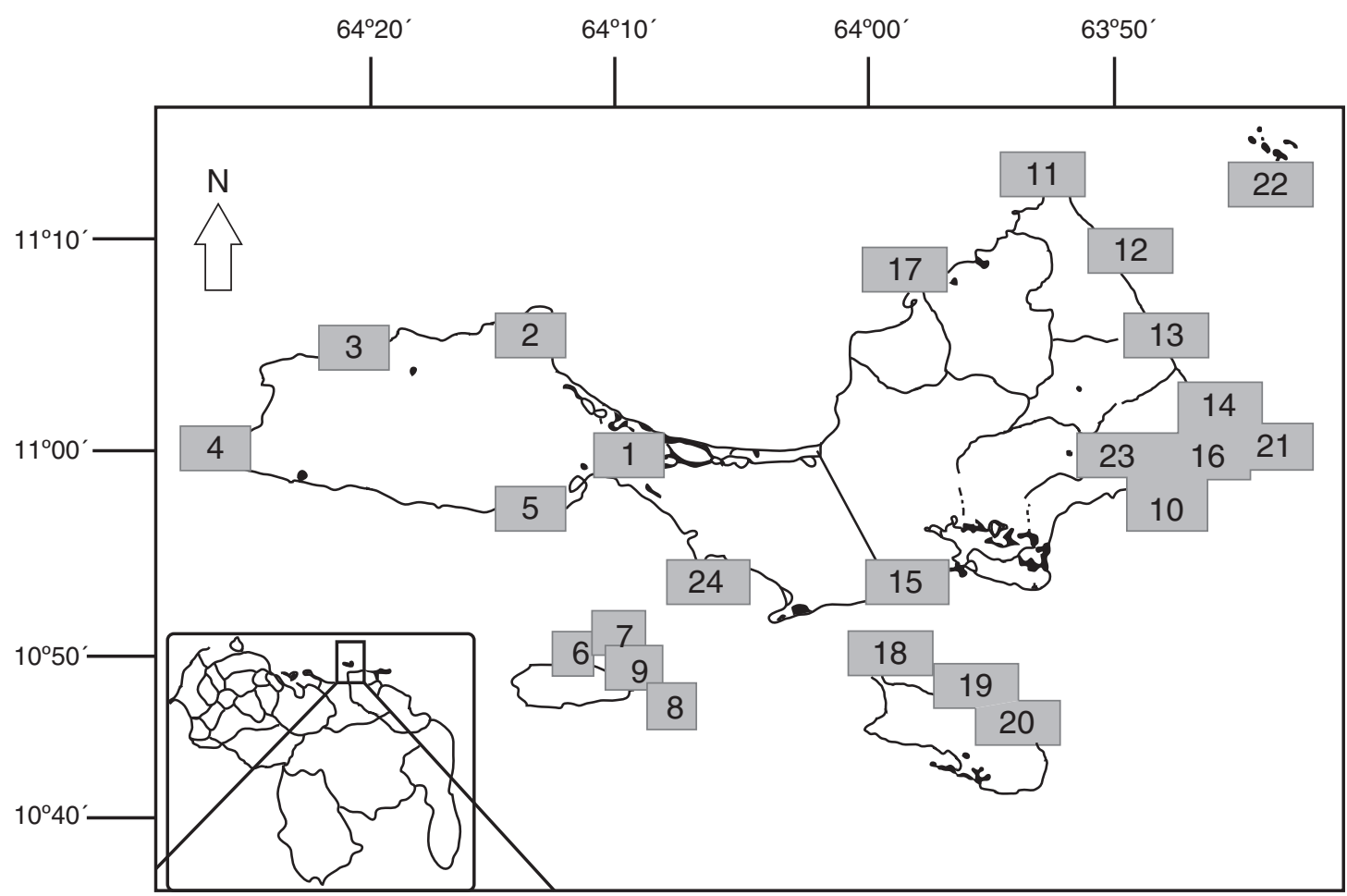

Figura 1. Mapa de Nueva Esparta donde se señalan los puntos de muestreo planteados. 1: Laguna de La Restinga; 2: El Maguey; 3: playa La Pared; 4: playa Punta Arena; 5: Boca del Río; 6: bahía de Charagato; 7: ferry hundido; 8: Las Cabeceras; 9: mercado; 10: playa Valdez; 11: Manzanillo; 12: playa El Agua; 13: playa Parguito; 14: punta Ballena; 15: playa El Yaque; 16: La Caranta; 17: playa Caribe; 18: bajo coche; 19: playa La Uva; 20: playa El Coco (coche); 21: El Farallón; 22: Infierno (Los Frailes); 23: laguna El Morro; 24: Punta de Piedras. 
Tabla 1

Especies de sacoglosos encontrados por localidad de muestreo.

\begin{tabular}{|c|c|c|}
\hline Localidad & Coordenadas & Especies \\
\hline La Restinga (1) & $\begin{array}{l}10^{\circ} 55^{\prime} 11.03^{\prime \prime} \mathrm{N} \\
64^{\circ} 01^{\prime} \mathrm{O}\end{array}$ & $\begin{array}{l}\text { E. serca, E. cauze, } \\
\text { L. souverbii, } \\
\text { O. antillarum, P. kingstoni }\end{array}$ \\
\hline Boca del Río (5) & $\begin{array}{l}10^{\circ} 58^{\prime} 5.62^{\prime \prime} \mathrm{N} \\
64^{\circ} 10^{\prime} 33.25^{\prime \prime} \mathrm{O}\end{array}$ & E. subornata \\
\hline Bahía de Charagato (6) & $\begin{array}{l}10^{\circ} 50^{\prime} 20.58^{\prime \prime} \mathrm{N} \\
64^{\circ} 9^{\prime} 24.88^{\prime \prime} \mathrm{O}\end{array}$ & E. subornata, $O$. antillarum \\
\hline Las Cabeceras (8) & $\begin{array}{l}10^{\circ} 49^{\prime} 25.97^{\prime \prime} \mathrm{N} \\
64^{\circ} 8^{\prime} 22.49^{\prime \prime} \mathrm{O}\end{array}$ & O. antillarum \\
\hline Playa Valdez (10) & $\begin{array}{l}10^{\circ} 56^{\prime} 50.67^{\prime \prime} \mathrm{N} \\
63^{\circ} 49^{\prime} 5.01^{\prime \prime} \mathrm{O}\end{array}$ & $\begin{array}{l}\text { E. serca, E. papillosa, } \\
\text { E. subornata, L. souverbii, } \\
\text { O. antillarum }\end{array}$ \\
\hline Playa El Yaque (15) & $\begin{array}{l}10^{\circ} 53^{\prime} 49.17^{\prime \prime} \mathrm{N} \\
63^{\circ} 57^{\prime} 27.27^{\prime \prime} \mathrm{O}\end{array}$ & E. serca \\
\hline Los Frailes (22) & $\begin{array}{l}11^{\circ} 11^{\prime} 30.50^{\prime \prime} \mathrm{N} \\
63^{\circ} 44^{\prime} 7.99^{\prime \prime} \mathrm{O}\end{array}$ & E. crispata \\
\hline
\end{tabular}

\section{Resultados}

Se encontraron un total de 7 especies pertenecientes a 3 familias de sacoglosos: Elysia serca Marcus, 1955, Elysia crispata (Mörch, 1863), Elysia subornata Verrill, 1901, Elysia cauze Marcus, 1957 (familia Plakobranchidae), Lobiger souverbii Fischer, 1856, Oxynoe antillarum Mörch, 1863 (familia Oxynoidae), Placida kingstoni Thompson, 1977 (familia Limapontiidae). Las especies E. subornata y E. cauze son consideradas sinónimos por Clark (1984) y Jensen (2007); sin embargo, las diferencias que presentaron los organismos recolectados nos permitieron identificarlas siguiendo el criterio de Marcus (1980), lo cual se explica posteriormente en el resumen taxonómico. Las especies se encontraron en 7 de los 24 puntos de muestreo (tabla 1), asociadas a diferentes sustratos (tabla 2).
Tabla 2

Especies de sacoglosos en función del sustrato donde fueron hallados.

\begin{tabular}{ll}
\hline Orden Sacoglossa & Sustrato \\
\hline Elysia serca & Thalassia testudinum \\
Elysia crispata & Piedras, algas verdes \\
Elysia subornata & Caulerpa sertularioides \\
Elysia cauze & Caulerpa sertularioides \\
Lobiger souverbii & Caulerpa sertularioides \\
Oxynoe antillarum & Caulerpa sertularioides \\
Placida kingstoni & Cladophora sp. \\
\hline
\end{tabular}

\section{Morfología externa}

Cuerpo alargado, de color crema con manchas marrones y verdosas. Rinóforos simples, traslúcidos con líneas verdes, lisos, cortos y con puntos blancos. Cerata largos y transparentes con una línea central verdosa con puntos blancos, verdes y marrones. En la cabeza se notan 2 líneas verdes a la altura de los ojos que se proyectan hacia los rinóforos. Ojos en la parte posterior de la cabeza. Zona pericárdica blanca con manchas marrones concentradas en ella. Cola corta con una línea verde. Pie estrecho, blanco cremoso y moteado.

\section{Morfología interna}

Rádula uniseriada estenoglosa, fórmula radular $22 \times 0.1 .0$, con todos los dientes ubicados en una cinta radular curvada. Diente radular liso y curvado. Cerata con el ducto de la glándula digestiva no ramificada y un comportamiento pulsante irregular. Órganos internos visibles por transparencia del cuerpo. La dermis produce una sustancia blanquecina como defensa química.

\section{Resumen taxonómico}

Material examinado. Cinco ejemplares 4.6, 5.2, 5.6, 7.7 y $8.7 \mathrm{~mm}$ de longitud (MOS007).

Clave para las especies de Sacoglossa encontradas en el presente estudio.

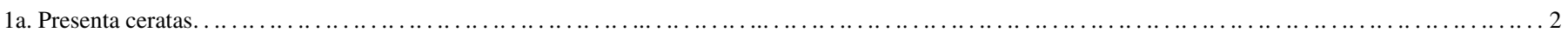

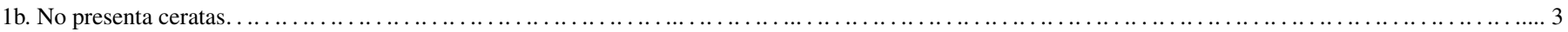

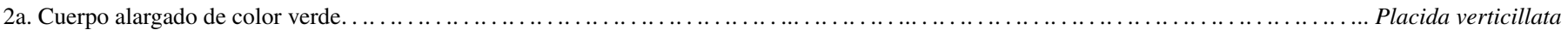

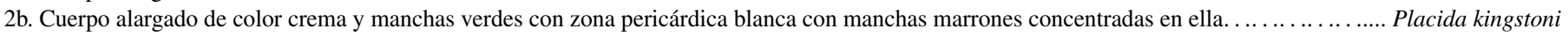

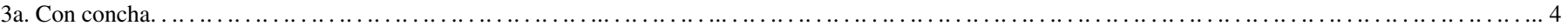

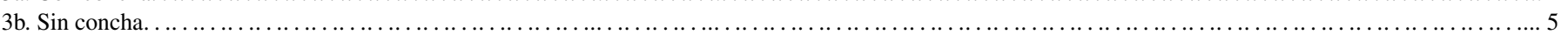

4a. Cuerpo alargado. Parapodios largos que cubren la concha hasta unirse en la porción posterior del dorso y en cuyos bordes poseen pequeñas

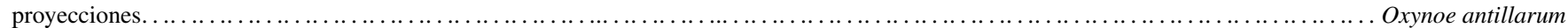

4b. Cuerpo ovalado. Concha cubierta por un manto con líneas negras irregulares. No presenta parapodios............................... Lobiger souverbii

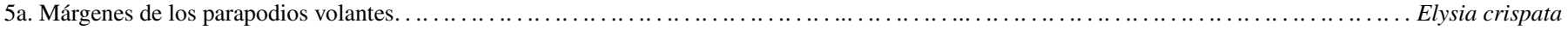

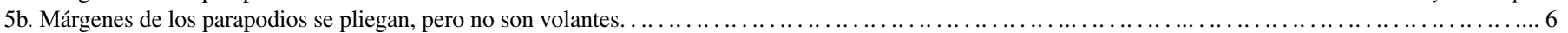

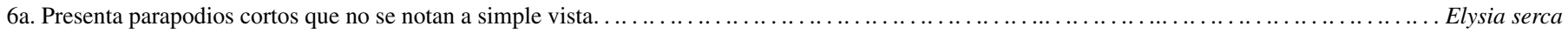

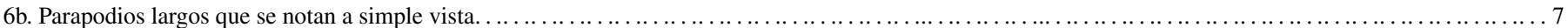

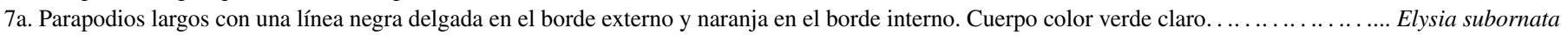

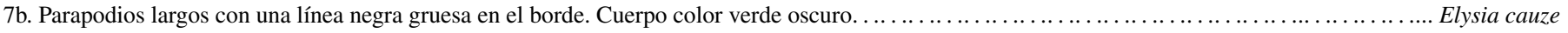

\section{Descripción}

Orden Sacoglossa Ihering, 1876

Familia Limapontiidae Gray, 1847

Placida kingstoni Thompson, 1977
Localidad y sustrato. Laguna La Restinga $\left(10^{\circ} 55^{\prime} 11.03^{\prime \prime} \mathrm{N}\right.$, $64^{\circ} 01^{\prime} \mathrm{O}$ ), a una profundidad de 0 a $1 \mathrm{~m}$, sobre Cladophora $\mathrm{sp}$.

Distribución. Bermudas, Costa Rica, Florida, Jamaica, Martinica (Valdés et al., 2006) y Venezuela (Grune et al., 2014). 


\section{Comentarios taxonómicos}

Transcurridos 3 días de la captura del animal, el color de este cambia de verde a marrón, al igual que el del alga en donde se encontró. Un detalle a destacar es que los cerata presentan un comportamiento pulsante tanto en el animal como al desprenderse del cuerpo. La descripción de este animal concuerda con la de Thompson (1977). Todos los ejemplares fueron encontrados en el alga verde filamentosa Cladophora sp.; sin embargo, también pueden ser hallados en algas del género Bryopsis. Suelen alimentarse del plasma celular, con la particularidad de procesar el líquido 3 veces antes de incorporarlo completamente al sistema digestivo (Thompson, 1977).

Familia Oxynoidae Stoliczka, 1868

Oxynoe antillarum Mörch, 1863

\section{Morfología externa}

Cuerpo alargado. Concha ovalada, con estrías de crecimiento, transparente, con un tinte verde manzana. Rinóforos simples, de color verde y con manchas blancas. Parapodios largos, verdes, cubriendo la concha; con bordes con pequeñas proyecciones blancas, tienden a unirse en la zona posterior del dorso. Palpos cortos. Pie estrecho y lobulado, de color blanco amarillento con círculos verde azulados a lo largo del borde, suele ser de color más claro que el resto del cuerpo. La cola representa más de la mitad de la longitud total del cuerpo y se caracteriza por presentar una banda de color blanco.

\section{Morfología interna}

Rádula uniseriada estenoglosa, fórmula radular $31 \times 0.1 .0$, con 10 dientes en la fila ascendente y 21 en la descendente, sin dientes en el ascus. Diente radular liso y recto que es usado para extraer el citoplasma de las ramas en forma de uva de Caulerpa.

\section{Resumen taxonómico}

Material examinado. Tres ejemplares de 2.4, 3.0 y $6.7 \mathrm{~mm}$ de longitud (MOSO02).

Localidad y sustrato. Laguna La Restinga $\left(10^{\circ} 55^{\prime} 11.03^{\prime \prime} \mathrm{N}\right.$, $\left.64^{\circ} 01^{\prime} \mathrm{O}\right)$, playa Valdez $\left(10^{\circ} 56^{\prime} 50.67^{\prime \prime} \mathrm{N}, 63^{\circ} 49^{\prime} 5.01^{\prime \prime} \mathrm{O}\right)$ y Las Cabeceras $\left(10^{\circ} 49^{\prime} 25.97^{\prime \prime} \mathrm{N}, 64^{\circ} 8^{\prime} 22.49^{\prime \prime} \mathrm{O}\right)$, a una profundidad de 0 a $1 \mathrm{~m}$, sobre el alga Caulerpa sertularioides.

Distribución. Florida, México, Honduras, Costa Rica, Panamá, Venezuela, Bahamas, Curazao, Bermudas, Jamaica, República Dominicana, Puerto Rico, Martinica, Barbados, Granada, Trinidad y Tobago (Valdés et al., 2006), y Guadalupe (Ortea et al., 2012).

\section{Comentarios taxonómicos}

La localización de los ejemplares fue igual a la descrita por Marcus y Hughes (1974). El color del animal descrito por el autor citado varía un poco con respecto al observado en el presente estudio, siendo de verde claro a oscuro, mientras que los encontrados solo presentaron un color verde claro. Los puntos encontrados en los rinóforos difirieron igualmente; el autor los describe como azules, mientras que los encontrados eran blancuzcos. La concha descrita en el trabajo citado, además, presentaba líneas negras en toda su longitud dorsal.

Lobiger souverbii Fischer, 1857

\section{Morfología externa}

Cuerpo ovalado de color verde y con puntos marrones. Rinóforos simples y largos y con la punta de color blanco. Concha dura, transparente, con una espira umbilical revestida por el manto. Manto verde con líneas negras irregulares. Cola corta. Parapodios con bordes ondulados. Cabeza con 2 pares de lóbulos orales.

\section{Morfología interna}

Rádula uniseriada estenoglosa, fórmula radular $12 \times 0.1 .0$, con 5 dientes en la fila ascendente, 7 en la descendente y numerosos en formación en el ascus. Diente radular liso y curvado. En el animal de mayor tamaño se contabilizaron 6 dientes en la hilera y 10 en el ascus.

\section{Resumen taxonómico}

Material examinado. Dos ejemplares de 3.7 y $5.5 \mathrm{~mm}$ de longitud (MOS006).

Localidad y sustrato. Playa Valdez $\left(10^{\circ} 56^{\prime} 50.67^{\prime \prime} \mathrm{N}\right.$, $63^{\circ} 49^{\prime} 5.01^{\prime \prime} \mathrm{O}$ ), a una profundidad de 0 a $1 \mathrm{~m}$, sobre el alga Caulerpa sertularioides.

Distribución. Barbados, Brasil, Curazao, Sri Lanka, Guadalupe, Estados Unidos de América, Puerto Rico, Costa Rica, México, Venezuela, Zanzíbar (Thompson, 1977; Valdés et al., 2006) y Guadalupe (Ortea et al., 2012).

\section{Comentarios taxonómicos}

La localización de los ejemplares coincide con la descrita por Marcus y Hughes (1974), quienes observaron la presencia de estos organismos en distintas especies de Caulerpa; sin embargo, estos autores apuntaron también al género Halimeda como hábitat del animal. El color difiere un poco del descrito por el autor, solo se encontraron individuos de color verde; sin embargo, en el trabajo citado se describieron organismos de color amarillo claro y verde claro, aunque los puntos marrones sí coincidieron con lo observado. Todas las otras características morfológicas descritas coinciden con lo observado.

Familia Plakobranchidae Gray, 1840

Elysia serca Marcus, 1955

\section{Morfología externa}

Cuerpo alargado. Rinóforos simples. Parapodios cortos y con manchas blancas en su zona media. Pie ancho. Cuerpo verde y con puntos blancos y negros a lo largo del dorso. En el interior de los parapodios se observan 8 vasos desiguales y algunos se encuentran unidos entre sí.

\section{Morfología interna}

Rádula uniseriada estenoglosa, fórmula radular $0 \times 1 \times 0$, con diente radular aserrado y curvado hacia dentro, con el extremo del borde cortante en ángulo recto. 
Distribución. Barbados, Brasil, Bonaire, Florida, Puerto Rico, San Martín y Curazao (Thompson, 1977; Valdés et al., 2006). Se registra por primera vez para Venezuela.

\section{Resumen taxonómico}

Material examinado. Cuatro ejemplares con rango de talla de 2.5 a 4 mm de longitud (MOS004).

Localidad y sustrato. Playa Valdez $\left(10^{\circ} 56^{\prime} 50.67^{\prime \prime} \mathrm{N}\right.$, $\left.63^{\circ} 49^{\prime} 5.01^{\prime \prime} \mathrm{O}\right)$, laguna La Restinga $\left(10^{\circ} 55^{\prime} 11.03^{\prime \prime} \mathrm{N}, 64^{\circ} 01^{\prime}\right.$ O) y playa El Yaque $\left(10^{\circ} 53^{\prime} 49.17^{\prime \prime} \mathrm{N}, 63^{\circ} 57^{\prime} 27.27^{\prime \prime} \mathrm{O}\right)$, a una profundidad de 0 a $1 \mathrm{~m}$, sobre Thalassia testudinum.

\section{Comentarios taxonómicos}

A simple vista la especie se diferencia de inmediato de las otras especies del género: los parapodios son muy cortos y es la única encontrada en Thalassia testudinum. A pesar de ser muy parecida a Elysia catulus, esta puede ser distinguida por el borde aserrado de la rádula.

Elysia subornata Verril, 1901

\section{Morfología externa}

Cuerpo alargado, con rinóforos que presentan proyecciones. Palpos bucales cortos con líneas marrones. Parapodios con una línea negra muy fina por el exterior y una línea naranja por el interior. Pie delgado y verde claro, amarillo en los bordes. Cuerpo verde claro con numerosas papilas, con puntos negros en toda su longitud y en los parapodios, los cuales, además, presenta proyecciones blanquecinas.

\section{Morfología interna}

Rádula uniseriada estenoglosa, fórmula radular $26 \times 0.1 .0$, con 5 dientes en la fila ascendente y 21 en la descendente, sin dientes en el ascus. Diente liso hasta su zona proximal, donde está ligeramente aserrado y curvado.

\section{Resumen taxonómico}

Material examinado. Cinco ejemplares con rango de tallas de 27 a $21.3 \mathrm{~mm}$ de longitud (MOS005).

Localidad y sustrato. Bahía de Charagato $\left(10^{\circ} 50^{\prime} 20.58^{\prime \prime} \mathrm{N}\right.$, $\left.64^{\circ} 9^{\prime} 24.88^{\prime \prime} \mathrm{O}\right)$, playa Valdez $\left(10^{\circ} 56^{\prime} 50.67^{\prime \prime} \mathrm{N}, 63^{\circ} 49^{\prime} 5.01^{\prime \prime}\right.$ O) y Boca del Río (10 $58^{\prime} 5.62^{\prime \prime} \mathrm{N}, 64^{\circ} 10^{\prime} 33.25^{\prime \prime} \mathrm{O}$ ), a una profundidad de 0 a $1 \mathrm{~m}$, sobre Caulerpa sertularioides.

Distribución. Florida, Puerto Rico, Jamaica, Islas Caimán, Bermudas, México, Venezuela, Antillas Menores, Trinidad y Tobago (Miloslavich et al., 2010; Valdés et al., 2006).

\section{Comentarios taxonómicos}

La especie, a primera vista, se parece mucho a su congénere E. cauze; sin embargo, el color de E. subornata es más claro y el cuerpo es más alargado, pero tal vez el elemento más importante en su identificación es el grosor de la línea negra que se encuentra en los extremos de los parapodios, así como la presencia de una línea naranja en el interior del parapodio (Marcus y Marcus, 1960). A su vez, se separa de Elysia ornata por la presencia de numerosas papilas a lo largo del cuerpo, ausentes en esta última.

Elysia cauze Marcus, 1957

\section{Morfología externa}

Cuerpo alargado, de color verde oscuro y con pequeñas manchas negras a los lados de este, aunque el color de fondo puede ser más claro en animales que viven en zonas con mucha iluminación. Rinóforos marrones con pequeños tubérculos blancos. Parapodios largos y verrugosos, presentan una línea negra gruesa en el borde.

\section{Morfología interna}

Rádula uniseriada estenoglosa, fórmula radular $23 \times 0.1 .0$, con 7 dientes en la fila ascendente, 16 en la descendente y numerosos en el ascus. Dientes lisos con el borde externo ligeramente aserrado.

\section{Resumen taxonómico}

Material examinado. Seis ejemplares con rango de talla de 15.4 a $31.2 \mathrm{~mm}$ de longitud (MOSO03).

Localidad y sustrato. Laguna La Restinga $\left(10^{\circ} 55^{\prime} 11.03^{\prime \prime} \mathrm{N}\right.$, $64^{\circ} 01^{\prime} \mathrm{O}$ ), a una profundidad de 0 a $1 \mathrm{~m}$, sobre algas verdes.

Distribución. Barbados, Brasil, Bonaire, Puerto Rico, San Martín, Florida (Thompson, 1977) y Guadalupe (Ortea et al., 2012). Se registra por primera vez para Venezuela.

\section{Comentarios taxonómicos}

Nuestros ejemplares concuerdan con la descripción dada por Thompson (1977), quien señala que la especie carece de tubérculos dentro de los parapodios. E. cauze es similar a E. subornata y fue sinonimizada por Clark (1984) con dicha especie. Jensen (2007) la considera como una especie inválida; sin embargo, encontramos diferencias como el color -es mucho más oscuroy el tamaño, menor en contraste con E. subornata. A su vez, Marcus y Marcus (1960) señalan que ambas especies se diferencian por la presencia de una línea negra en el borde externo del parapodio y una línea naranja en el borde interno en $E$. subornata, esta última ausente en $E$. cauze, la cual pudo observarse claramente en los ejemplares recolectados, coincidiendo con las descripciones realizadas por Marcus (1980) y Marcus y Hughes (1974). Ortea et al. (2013) señalan las dificultades que existen en la identificación de las especies de Elysia en el Caribe, y afirman durante su revisión que algunas de las especies identificadas en Bahamas, como, por ejemplo, E. subornata,son en realidad E. cauze y Elysia nisbeti. E. cauze se diferencia de $E$. nisbeti, especie descrita por Thompson (1977), por la presencia de una marca en forma de $\mathrm{Y}$ en la cabeza y por la presencia de líneas negras en lugar de ocelos en los flancos. Marcus y Hughes (1974) registran la especie solamente asociada a Caulerpa.

Elysia crispata (Mörch, 1863) 


\section{Morfología externa}

Cuerpo alargado. Rinóforos lisos y contráctiles. Palpos bucales de considerable tamaño. Parapodios plegados en toda la extensión del dorso, llegando a representar la mitad de la altura del cuerpo. Rinóforos y parapodios con un margen blanco, delgado y seguido de una banda verde o azul y crema.

\section{Morfología interna}

Rádula uniseriada estenoglosa, fórmula radular $17 \times 0.1 .0$, con 9 dientes en la fila ascendente, 8 en la descendente y 25 en el ascus. Diente radular ligeramente aserrado.

\section{Resumen taxonómico}

Material examinado. Diez ejemplares con rango de talla de 10 a $53.5 \mathrm{~mm}$ de longitud (MOS001).

Localidad y sustrato. Los Frailes $\left(11^{\circ} 11^{\prime} 30.50^{\prime \prime} \mathrm{N}\right.$, $63^{\circ} 44^{\prime} 7.99^{\prime \prime} \mathrm{O}$ ), a una profundidad de 1 a $4 \mathrm{~m}$, sobre piedras.

Distribución. Florida, México, Honduras, Costa Rica, Colombia, Aruba, Cuba, Islas Caimán, Curazao, Bonaire, Bermudas, Bahamas, Jamaica, Haití, Puerto Rico, Barbados, Trinidad y Tobago, Venezuela (Miloslavich et al., 2010; Valdés et al., 2006) y Guadalupe (Ortea et al., 2012).

\section{Comentarios taxonómicos}

Nuestros ejemplares se adaptan a los caracteres descritos por Thompson (1977). La profundidad a la que viven los ejemplares parece estar relacionada con los tamaños hallados, encontrando los más pequeños a $1 \mathrm{~m}$ y los más grandes a $4 \mathrm{~m}$. Marcus y Hughes (1974) señalan que la especie presenta 2 patrones de coloración: verde con crema o azul con crema, coincidiendo ambos con los colores presentados por los ejemplares encontrados. Esta especie fue descrita originalmente dentro del género Tridachia Deshayes, 1857, pero fue transferida al género Elysia por Mörch, 1863. En el campo son fácilmente reconocibles por sus exuberantes parapodios de llamativos colores.

\section{Referencias}

Ardila, N., Báez, D. y Valdés, A. (2007). Babosas y liebres de mar (Mollusca: Gastropoda: Opisthobranchia) de Colombia. Biota Colombiana, 8, 185-197.

Caballer, M. (2007). Catálogo de los sacoglosos y opistobranquios (Mollusca: Gastropoda) de sustrato rocoso de la bahía de Santander. Revisión de los géneros con especies crípticas. Tesis doctoral. Santander: Universidad de Cantabria.

Caballer, M. y Ortea, J. (2012). Description of a new species of Hypselodoris (Gastropoda: Nudibranchia: Chromodorididae) from Venezuela. Revista de la Academia Canaria de Ciencias, 23, 93-106.

Caballer, M. y Ortea, J. (2015). A new shallow water species of the genus Philine Ascanius, 1772 (Mollusca: Opisthobranchia: Philinidae) from Venezuela. Journal of Conchology, 42, 1-8.

Caballer, M., Ortea, J. y Espinosa, J. (2006). Descripción de una nueva especie de Alderiopsis Baba, 1968. En Moluscos marinos de la península de Guanahacabibes, Pinar del Río, Cuba, con la descripción de nuevos taxones. Avicennia, 18, 57-59.

Capelo, J. y Buitrago, J. (1998). Distribución geográfica de los moluscos marinos en el oriente de Venezuela. Memoria - Sociedad de Ciencias Naturales La Salle, LVIII, 109-160.

Capelo, J., Rada, M., Solé, M., Buitrago, J., Grune, S. y Narváez, J. (2014). Los moluscos marinos litorales del norte de la península de Macanao, isla de Margarita, Venezuela. Amici Molluscarum, 22, 29-44.
Cimino, G. y Ghiselin, M. (1999). Chemical defense and evolutionary trends in biosynthetic capacity among dorid nudibranchs (Mollusca: Gastropoda: Opistobranchia). Chemoecology, 9, 187-207.

Clark, K. (1984). New records and synonymies of Bermuda opisthobranchs (Gastropoda). Nautilus, 98, 85-97.

Crescini, R., de Sisto, M. y Villalba, W. (2013). A new species of nudibranch of the genus Learchis (Gastropoda: Heterobranchia: Facelinidae) from the tropical western Atlantic Ocean. American Malacological Bulletin, 31, 339-341.

Crescini, R., Villalba, W. y de Sisto, M. (2013). Primer registro de Doto chica (Mollusca: Dotidae) en Venezuela. Revista Mexicana de Biodiversidad, 84, 668-670.

De Sisto, M. (2014). Heterobranquios (Mollusca: Gastropoda) del estado Nueva Esparta, Venezuela. Tesis. Boca del Río, Venezuela: Universidad de Oriente.

Edmunds, M. (1964). Eolid mollusca from Jamaica, with descriptions of two new genera and three new species. Bulletin of Marine Science of the Gulf and Caribbean, 14, 1-32.

Edmunds, M. y Just, H. (1983). Eolid nudibranchiate mollusca from Barbados. Journal of Molluscan Studies, 49, 185-203.

Gavagnin, M., Mollo, E., Castelluccio, F., Montanaro, D., Ortea, J. y Cimino, G. (1997). A novel dietary sesquiterpene from the marine sacoglossan Tridachia crispata. Natural Product Letters, 10, 151-156.

Gavagnin, M., Mollo, E., Cimino, G. y Ortea, J. (1996). A new gammadihydropyrone-propionate from the Caribbean sacoglossan Tridachia crispata. Tetrahedron Letters, 37, 4259-4263.

Gavagnin, M., Mollo, E., Montanaro, D., Ortea, J. y Cimino, G. (2000). Chemical studies on Caribbean sacoglossans: dietary relationships with green algae and ecological implications. Journal of Chemical Ecology, 26, $1563-1578$

Grune, S., Crescini, R., de Sisto, M., Velásquez, M. y Villalba, W. (2014). Opistobranquios del Parque Nacional Laguna de La Restinga, Isla de Margarita, Venezuela. Amici Molluscarum, 22, 25-35.

Jensen, K. (1997). Evolution of the Sacoglossa (Mollusca, Opisthobranchia) and the ecological associations with their food plants. Evolutionary Ecology, 11, 301-335.

Jensen, K. (2007). Biogeography of the Sacoglossa (Mollusca Opisthobranchia). Bonner Zoologische Beiträge, 55, 255-281.

Maeda, T., Kajida, T., Maruyama, T. y Hirano, Y. (2010). Molecular phylogeny of the sacoglossa, with a discussion of gain and loss of kleptoplasty in the evolution of the group. Biological Bulletin, 219, 17-26.

Marcus, E. (1980). Review of western Atlantic elysiidae (Opisthobranchia Ascoglossa) with a description of a new Elysia species. Bulletin of Marine Science, $30,54-79$.

Marcus, E. y Hughes, H. (1974). Opisthobranchs mollusks from Barbados. Bulletin of Marine Science, 24, 498-532.

Marcus, E. y Marcus, E. (1960). Opisthobranchs from American Atlantic warm waters. Bulletin of Marine Science of the Gulf and Caribbean, 10, 129-203.

Marcus, E. y Marcus, E. (1967). Opisthobranchs from the southwestern Caribbean Sea. Bulletin of Marine Science, 17, 597-628.

Mariño, J., Farfan, E. y Caballer, M. (2011). Primer registro de Dondice parguerensis (Mollusca: Favorinidae) para Venezuela. Revista Mexicana de Biodiversidad, 82, 709-712.

Mikkelsen, P. (2002). Advances in marine biology (42) London: Academic Press.

Miloslavich, P., Díaz, J., Klein, E., Alvarado, J., Díaz, C., Gobin, J., et al. (2010). Marine biodiversity in the Caribbean: regional estimates and distribution patterns. PloS One, 5, 1-25.

Ortea, J. y Espinosa, J. (2002). Nuevas especies del género Elysia Risso, 1818 (Mollusca: Sacoglossa) con caracteres singulares. Avicennia, 15, 129-140.

Ortea, J., Espinosa, J., Buske, Y. y Caballer, M. (2013). Additions to the inventory of the sea slugs (Opisthobranchia and Sacoglossa) from Guadeloupe (Lesser Antilles, Caribbean Sea). Revista de la Academia Canaria de Ciencia, XXV, 163-194.

Ortea, J., Espinosa, J., Caballer, M. y Buske, Y. (2012). Initial inventory of the sea slugs (Opisthobranchia and Sacoglossa) from the expedition Karubenthos, held in May 2012 in Guadeloupe (Lesser Antilles, Caribbean Sea). Revista de la Academia Canaria de Ciencias, 24, 153-182. 
Ortea, J., Moro, L., Bacallado, J. y Espinosa, J. (1998). Catálogo abreviado de las especies del orden Sacoglossa (=Ascoglossa, Mollusca: Opisthobranchia) de las Islas Canarias y de Cabo Verde. Revista de la Academia Canaria de Ciencias, 10, 85-96.

Ortea, J., Moro, L. y Espinosa, J. (1997). Nuevos datos sobre el género Elysia Risso, 1818 (Opisthobranchia: Sacoglossa) en el Atlántico. Revista de la Academia Canaria de Ciencias, 9, 141-155.
Thompson, T. (1977). Jamaican opisthobranch molluscs. Journal of Molluscan Studies, 43, 93-140.

Thompson, T. (1980). Jamaican opisthobranch molluscs II. Journal of Molluscan Studies, 46, 74-99.

Valdés, A., Hamann, D. y Dupont, A. (2006). Caribbean sea slugs. Washington, D. C.: Sea Challengers Natural History Books. 